\title{
SISTEMA DE IRRIGAÇÃO AUTOMÁTICA COM ARDUINO
}

\author{
Amanda Rufino Matricciani ${ }^{1}$ \\ Davi da Silva Nogueira ${ }^{2}$ \\ Gustavo de Aquino Lima $^{3}$ \\ Lucas Cortez Marcolino 4 \\ Nichollas Bartho Oliveira Santos ${ }^{5}$ \\ Rafael Cesar Barboza ${ }^{6}$ \\ Rafael Hajime Ishikawa ${ }^{7}$ \\ Rodrigo Izumi Braga Ueno ${ }^{8}$ \\ Amanda Sant' Anna Sciammarella Montecin ${ }^{9}$
}

Resumo: Observa-se nas grandes cidades uma crescente criação de hortas caseiras, para assim obter hortaliças frescas e de boa qualidade. Mas nem todas as pessoas têm tempo para cuidar de sua horta. Para minimizar este problema foi proposta uma forma de regar as plantas de forma automatizada, sem que o proprietário tenha que se preocupar. Através do uso do Arduino Uno, sensores e bomba d'água foi possível criar um sistema que mede a umidade do solo em tempo real e irriga quando necessário, visto que cada planta tem um valor de umidade específico, evitando desperdícios como analisado nos testes. Aliado a este fato, o uso de materiais de baixo custo contribui tornando o projeto mais viável e sustentável, entretanto este abrange ainda uma pequena área para cultivo caseiro.

Palavras-chave: Arduino Uno; Irrigação; Automático; Sustentabilidade; Sensores.

\footnotetext{
1 Programa Pré Universitário Pré Exatas/Colégio Embraer Juarez Wanderley, Brasil. E-mail: amanda.matricciani@outlook.com.

2 Programa Pré Universitário Pré Exatas/Colégio Embraer Juarez Wanderley, Brasil. E-mail: davinogueira_00@hotmail.com.

3 Programa Pré Universitário Pré Exatas/Colégio Embraer Juarez Wanderley, Brasil. E-mail: gustavoaquinodelima@gmail.com.

4 Programa Pré Universitário Pré Exatas/Colégio Embraer Juarez Wanderley, Brasil. E-mail: lucas.cortezz@hotmail.com.

5 Programa Pré Universitário Pré Exatas/Colégio Embraer Juarez Wanderley, Brasil. E-mail: n.bartho@yahoo.com.

6 Programa Pré Universitário Pré Exatas/Colégio Embraer Juarez Wanderley, Brasil. E-mail: rafael_cb27@hotmail.com.

7 Programa Pré Universitário Pré Exatas/Colégio Embraer Juarez Wanderley, Brasil. E-mail: rafael.ishikawa00@gmail.com.

8 Programa Pré Universitário Pré Exatas/Colégio Embraer Juarez Wanderley, Brasil. E-mail: rodrigo.izumi@hotmail.com.

${ }^{9}$ Colégio Embraer Juarez Wanderley, Brasil. E-mail: mandy_santanna@hotmail.com.
} 